\title{
Prognosis in people with back pain
}

\author{
Rachelle Buchbinder MBBS PhD, Martin Underwood MD
}

See related research article by Costa and colleagues at www.cmaj.ca/lookup/doi/10.1503/cmaj.111271

$\mathrm{L}$ ow-back pain has a large personal and societal impact. Most episodes of back pain are mild and short-lived, and only a few people (i.e., about 1 in 20) seek care. ${ }^{1}$ It is the small minority of these people, who develop chronic disabling back pain, that account for the economic and social costs of back pain. In a related article, Costa and colleagues have made two important contributions to our understanding of the clinical course of low-back pain by performing a systematic review of prospective studies that enrolled episode-inception cohorts. ${ }^{2}$ First, they identified the similarities and differences in one-year outcomes among people who presented with either acute or persistent pain. Second, they collated what is known about prognostic markers in low-back pain.

Prognostic studies can be broadly classified into four groups: those that describe the course of the condition over time, those that aim to identify factors associated with or that determine the outcome, those that aim to identify subgroups of people who may have different outcomes, and those that test the impact of predictive modelling. ${ }^{3}$ The review by Costa and coauthors, which focuses on the first type of prognostic study, included 33 episode-inception cohorts of back pain involving 11166 participants. The cohorts were divided on the basis of episode duration: acute low-back pain cohorts were defined as mean or median pain duration of less than six weeks at study entry, and persistent low-back pain cohorts were defined as mean or median pain of six or more weeks at study entry. ${ }^{2}$ They found that improvement within the first six weeks (from either study entry or from a time adjusted for symptom duration) was rapid in both the acute and persistent groups. By 12 months those who presented with a short history of pain typically had minimal pain and disability, while those who had a longer duration of pain at the time of presentation continued to have low to moderate levels of pain but with more residual disability.

An important conclusion that can be drawn from their data is that much of the apparent benefits of any treatment for back pain are likely to be unrelated to the effects of specific interventions. Although the authors excluded data from randomized controlled trials on the basis that their inclusion might limit the generalizability of their results, it is noteworthy that the general pattern of improvement over time (rapid initial improvement followed by a plateau) mirrors what has been observed in randomized controlled trials for a wide range of primary care treatments for low-back pain, irrespective of the treatment arm. ${ }^{4}$ Similar to the systematic review of randomized controlled studies by Artus and colleagues, ${ }^{4}$ Costa and colleagues found moderate to high between-person and between-study heterogeneity, indicating the presence of wide variability in outcomes both within and across studies. Although Costa and colleagues performed subgroup analysis on the basis of the duration of symptoms, other subgroup analyses (e.g., by setting) might have been able to explain some of the observed heterogeneity.

As the authors acknowledged, between-study heterogeneity may also have been due to different risks of bias among the included studies. Important potential sources of bias in prognostic studies include the representativeness of the study sample for the population of interest, the degree of study attrition, the adequacy of measurement of prognostic factors, outcomes and potential confounders, and the adequacy of the statistical analysis. ${ }^{5}$ Although Costa and colleagues considered some of these as part of their quality appraisal of the included studies, none were incorporated into their synthesis of evi-

\section{KEY POINTS}

- Most people who seek care for low-back pain improve rapidly within the first six weeks of presentation.

- In contrast to people with a shorter duration of symptoms when they seek care, people with more persistent pain may still have moderate levels of disability at one year.

- Much of the apparent benefits of treatment for back pain are likely unrelated to the effects of specific interventions.

- Future research should investigate what contributes to residual disability from an episode of low-back pain and identify better treatment targets. 
dence. All reviews of prognostic studies are recommended to perform sensitivity analyses to assess the robustness of their results to bias. ${ }^{5}$ One approach might be to perform separate sensitivity analyses for different important biases, as well as a sensitivity analysis omitting all studies that are at high risk of any important bias. ${ }^{5}$

A striking finding from this review is the difference in prognosis for pain and disability in the persistent low-back pain cohort. Although, as one might expect, both pain and disability improved rapidly in the acute pain cohorts, this was not the case in the persistent pain cohorts. In this cohort, disability improved slowly, matching the anticipated pattern of poor prognosis. Pain, however, improved substantially with time, albeit more slowly than in the acute pain cohort. That disability, rather than low-back pain, persists is an important pointer for improving our interventions for back pain, and this argues for rethinking our terminology. There is little point in targeting pain when, in fact, it is disability that is the problem.

Understanding what contributes to the continued disability and ensuring that it is adequately measured and considered will go a long way toward identifying better treatment targets. Disempowerment, loss of independence, worry about the future, perceived or real negative or discriminatory actions by others, and secondary health effects have all been identified as important contributors to the burden of back pain, ${ }^{6}$ yet these are not currently captured in outcome studies.

Costa and colleagues also extracted data about prognostic factors from all cohorts, although significant variability in domains studied and analytic approaches precluded pooling of the data or exploration of prognostic factors across subgroups. These methodologic issues have previously been identified, and future cohort studies of low-back pain should use the Multinational Musculoskeletal Inception Cohort Study Statement standardized core explanatory variables for inception cohorts to facilitate data pooling and comparison across different health care settings. ${ }^{7}$ A previous study that combined data from two large prospective cohort studies of patients in primary care found that, apart from fear of pain, which was a significant predictor of disability at 12 months only among patients with back pain of greater than 3 months duration, the prognostic indicators did not differ on the basis of symptom duration at presentation. ${ }^{8}$

These and other data have contributed to a slow paradigm shift toward viewing back pain as a chronic recurring condition, replacing more traditional classifications based on the duration of symptoms. More frequent and longer follow up of individuals with back pain has revealed the existence of distinct clusters that behave differently over time. ${ }^{9}$ Further research defining clinically meaningful subgroups of people based on their individual course of back pain over time may also lead to important advances in the management of low-back pain.

\section{References}

1. Vingård E, Mortimer M, Wiktorin C, et al. Seeking care for low back pain in the general population: a two-year follow-up study: results from the MUSIC-Norrtälje Study. Spine 2002;27:2159-65.

2. Costa D, Maher C, Hancock M, et al. The prognosis of acute and persistent low back pain: a meta-analysis. CMAJ 2012;184:E613-24.

3. Hayden JA, Dunn K, van der Windt D, et al. What is the prognosis of back pain? Best Pract Res Clin Rheumatol 2010;24:167-79.

4. Artus M, van der Windt DA, Jordan KP, et al. Low back pain symptoms show a similar pattern of improvement following a wide range of primary care treatments: a systematic review of randomized clinical trials. Rheumatol (Oxford) 2010;49:2346-56.

5. Hayden JA, Cote P, Bombardier C. Evaluation of the quality of prognosis studies in systematic reviews. Ann Intern Med 2006; 144:427-37.

6. Buchbinder R, Batterham R, Elsworth G, et al. A validity-driven approach to the understanding of the personal and societal burden of low back pain: development of a conceptual and measurement model. Arthritis Res Ther 2011:13:R152.

7. Pincus T, Santos R, Breen A, et al. Multinational Musculoskeletal Inception Cohort Study Collaboration. A review and proposal for a core set of factors for prospective cohorts in low back pain: a consensus statement. Arthritis Rheum 2008;59:14-24.

8. Grotle M, Foster NE, Dunn KM, et al. Are prognostic indicators for poor outcome different for acute and chronic low back pain consulters in primary care? Pain 2010;151:790-7.

9. Dunn Km, Jordan K, Croft P. Characterizing the course of low back pain: a latent class analysis. Am J Epidemiol 2006;163:754-61.

Affiliations: From the Monash Department of Clinical Epidemiology (Buchbinder), Cabrini Hospital; the Department of Epidemiology and Preventive Medicine (Buchbinder), School of Public Health and Preventive Medicine, Monash University, Malvern, Australia; and the Division of Health Sciences (Underwood), Warwick Medical School, the University of Warwick, Coventry, UK.

Contributors: Both authors drafted the commentary and approved the final version submitted for publication. 